\title{
Variations saisonnières des proportions relatives des acides gras d'un beurre de laiterie d'Ille-et-Vilaine
}

\author{
par \\ J. P. GALLACIER* , J. P. BARBIER* \\ et S. KUZDZAL-SAVOIE** \\ I. - INTRODUCTION
}

Diverses études poursuivies en France et à l'étranger au cours des années 1950-1960 ont mis en évidence l'existence de variations saisonnières de l'indice d'iode des beurres fabriqués dans différentes régions de France ou dans différents pays. Un indice d'iode plus élevé en période de pâturage qu'en période de stabulation est généralement observé dans les régions ou pays où le cheptel bovin est soumis à l'alternance de ces deux modes de vie. Dans les régions où les vaches laitières restent au pâturage toute l'année (en NouvelleZélande par exemple, comme d'ailleurs dans certaines parties de la Normandie française) l'amplitude de variations de l'indice d'iode du beurre est relativement faible et parfois même un indice d'iode minimum est observé pendant les mois d'été (Cf. Béjambes et Savoie, 1954).

De très nombreux travaux ont été consacrés à l'étude des variations de l'indice d'iode du beurre. L'alimentation des vaches laitières et le stade de lactation constituent les facteurs dont l'influence est prépondérante.

Les variations de l'indice d'iode du beurre ne sont que le reflet des variations qui atteignent la composition des acides gras du beurre puisque les triglycérides représentent au minimum 98 p. 100 de la matière grasse laitière totale.

En 1952, Hansen et Shorland, utilisant la technique de distillation fractionnée, ont déterminé les proportions relatives des acides gras

\footnotetext{
* Laiteries E. Bridel - 35240 Retiers (France).

** Station Centrale de Recherches Laitières et de Technologie des Produits Animaux, I.N.R.A. - 78350 Jouy-en-Josas (France).
} 
majeurs du beurre de Nouvelle-Zélande au cours d'une année. Mais, précisément à cette date (James et Martin, 1952), apparurent les premières applications de la chromatographie en phase gazeuse dans le domaine de l'analyse des lipides, et cette nouvelle technique se révéla aussitôt un outil particulièrement bien adapté à l'analyse des acides gras du beurre.

De 1960 à 1972, furent ainsi publiés de multiples travaux consacrés à l'étude détaillée des acides gras du beurre (identification des acides gras mineurs et estimation des proportions relatives). Des milliers d'échantillons de beurre d'origines très diverses, correspondant à des prélèvements répartis sur une ou sur plusieurs années furent analysés dans de nombreux pays.

Ces études constituent une incontestable nouveauté en ce qui concerne le développement des connaissances relatives aux acides gras du beurre, et en ce qui concerne les moyens mis ainsi à la disposition des analystes en vue de contrôler la pureté du beurre.

Un bref rappel des publications consacrées à l'étude des variations saisonnières de composition des acides gras du beurre sera présenté avant d'exposer en détail les résultats d'une étude ponctuelle effectuée au cours de l'année 1972 sur le beurre fabriqué à partir du lait collecté dans le sud de l'Ille-et-Vilaine.

Dès 1960, Patton et al. comparèrent la composition des acides gras des beurres d'été et des beurres d'hiver fabriqués dans une région des Etats-Unis.

A cette même époque, des chercheurs italiens (de Francesco et al., 1961) publièrent de très nombreux résultats d'analyse par chromatographie en phase gazeuse des acides gras du beurre fabriqué dans différentes régions d'Italie. Les travaux italiens ne cessèrent de se développer (Strocchi et al., 1967, Dreassi et al., 1970).

L'analyse des acides gras des beurres hollandais fut entreprise par les chercheurs de la Station de Contrôle d'Etat (Roos et al., 1963).

Guyot et Piraux, en Belgique, publièrent en 1964 et en 1965 les résultats d'analyses effectuées sur des beurres fabriqués dans différentes régions de Belgique et exploitèrent ces résultats en vue de combattre certaines formes nouvelles de falsification du beurre (Guyot, 1968). Huyghebaert et Hendrickx développèrent ces travaux (1970, 1971).

En Norvège (Svensen et Ystgaard, 1966), en Grande-Bretagne (Hutton et al., 1969 ; Hall, 1970), en Irlande (Richardson et McGanri, 1964), en Suède (Mattsson et al., 1969), en Allemagne (Metin, 1968 ; Senft et al., 1970), en Suisse même (Hadorn, 1969, 1970), des travaux analogues furent entrepris.

Plus récemment (Olmedo et al., 1971) l'étude des acides gras du beurre fabriqué dans diverses régions d'Espagne fit l'objet d'une publication très détaillée. Des pays plus lointains tels la 
Pologne (Budslawski et al., 1969), l'Australie (Parodi, 1970, 1972) fournirent également des informations précieuses sur la composition des acides gras du beurre.

En France, aucune étude systématique n'a été jusqu'ici publiée, bien que de très nombreuses analyses aient été effectuées ces dernières années sur des échantillons d'origines variées.

C'est pourquoi, il a semblé intéressant de présenter les résultats obtenus lors de l'analyse par chromatographie en phase gazeuse des acides gras du beurre fabriqué dans une entreprise laitière.

\section{II. - PARTIE EXPERIMENTALE}

\section{1) Origine des échantillons et fréquence des analyses}

Le beurre fabriqué à partir du lait ramassé dans la région de Retiers en Ille-et-Vilaine a été analysé régulièrement de janvier 1972 à janvier 1973, chaque semaine. Toutefois, seules les moyennes mensuelles établies à partir des résultats des analyses hebdomadaires ont été considérées.

\section{2) Analyses des acides gras}

\section{a) ESTÉRIFICATION}

Les acides gras des triglycérides ont été transformés en esters méthyliques par transestérification en milieu acide, dans une ampoule scellée, selon une technique voisine de celle utilisée par Huyghebaert et Hendrickx (1970 b).

«Dans un tube en pyrex de $5 \mathrm{ml}$ préalablement étiré, introduire successivement $2 \mathrm{~g}$ de matière grasse séchée, puis $0,4 \mathrm{ml}$ d'une solution méthanolique d'acide sulfurique à 3 p. $100(\mathrm{p} / \mathrm{p})$. Sceller le tube et le maintenir $2 \mathrm{~h}$ à $100^{\circ} \mathrm{C}$. Après refroidissement, l'ampoule est ouverte, et quelques $\mu \mathrm{l}$ de la phase supérieure sont injectés dans le chromatographe».

b) ANalyse des esters par chromatographie en pHaSe gazeuse

\section{- Appareillage}

Un chromatographe Girdel 3000 , équipé de deux détecteurs à ionisation de flamme, a été utilisé. Un enregistreur Servoriter II - Texas - et un intégrateur électronique - chromalog 2 Kent ont complété l'équipement.

\section{- Choix de la phase stationnaire}

Lors de l'analyse des acides gras du beurre, trois types de phases stationnaires polaires sont généralement utilisés : succinate d'éthy- 
lène glycol, succinate de diéthylène glycol et succinate de butane diol ou polyester de Craig. Mais, beaucoup d'autres phases conviennent également (Carbowax $20 \mathrm{M}$, EGSSX, etc.).

Le succinate de diéthylène glycol permet une analyse rapide des acides majeurs du beurre et une bonne résolution du couple acide stéarique / acide oléique. Depuis plusieurs années, S. Kuzdzal (Kuzdzal et al., 1967, 1969, 1970) préconise cependant - et bien que la durée de l'analyse soit accrue - l'utilisation de succinate de butane diol pour l'analyse des acides gras du beurre. En effet, cette phase stationnaire permet une bonne résolution des acides ramifiés et des acides monoènes comptant un atome de carbone de moins, et, en outre, permet de distinguer sur le chromatogramme, le pic correspondant à l'acide diène conjugué et le pic correspondant à l'acide linolénique.

Une colonne en acier inoxydable d'une longueur de $3 \mathrm{~m}$ et d'un diamètre extérieur de $0,35 \mathrm{~cm}$, chargée de chromosorb W lavé aux acides et imprégné de succinate de butane diol au taux de 20 p. 100 a été utilisée.

\section{- Conditions de l'analyse}

Quelques analyses ont été effectuées dans des conditions de température programmée : accroissement de $2^{\circ} \mathrm{C}$ par mn entre $80^{\circ} \mathrm{C}$ et $130^{\circ} \mathrm{C}$ suivi d'un accroissement de $0,5^{\circ} \mathrm{C}$ par mn jusqu'à $200^{\circ} \mathrm{C}$. Malheureusement, cette méthode, délicate quant aux réglages, accroît considérablement la durée des analyses. Très généralement donc des conditions isothermiques d'analyse ont été adoptées $\left(200^{\circ} \mathrm{C}\right)$. Une pression d'entrée de 1,7 bar et un débit de $15 \mathrm{ml} / \mathrm{mn}$ ont été choisis pour le gaz vecteur (azote).

Les surfaces relatives des pics ont été calculées à l'aide de l'intégration électronique. L'utilisation de la relation logarithme du temps de rétention (par rapport à l'acide stéarique) et nombre d'atomes de carbone, jointe à l'examen des résultats obtenus antérieurement par divers auteurs, a permis d'obtenir une identification très probable sinon absolue des pics observés sur les chromatogrammes.

\section{III. - RESULTATS}

\section{1) Analyse chromatographique}

Le chromatogramme représenté sur la figure 1 a été obtenu dans des conditions de température programmée. Cette méthode nous a permis d'obtenir 35 pics bien résolus.

Les figures 2 et 3 constituent une illustration des chromatogrammes obtenus dans des conditions isothermiques d'analyse. Dans ces conditions, les surfaces respectives de 29 pics d'acides gras ont pu être calculées. 


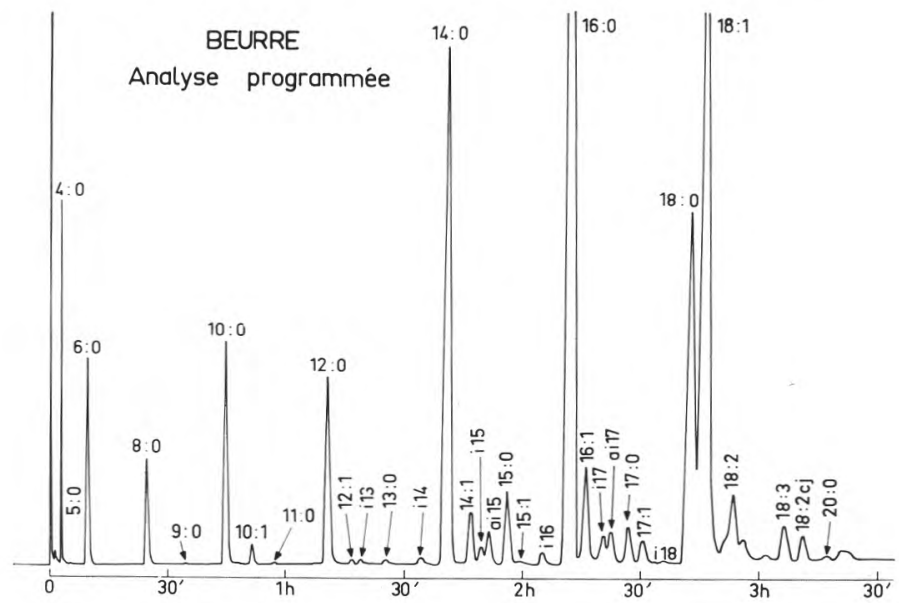

fig. 1

Analyse par chromatographie en phase gazeuse des acides gras du beurre de Retiers.

Conditions de l'analyse : Chromatographe Girdel 3000, équipé d'un détecteur à ionisation de flamme ; colonne en acier inoxydable de $3 \mathrm{~m}$ de long et de $0,3 \mathrm{~cm}(1 / 8 \mathrm{~s})$ de diamètre intérieur, remplie de chromosorb W, lavé aux acides (80-100 mesh) imprégné de succinate de butane diol au taux de 20 p. 100 ; programmation de température : de $80^{\circ} \mathrm{C}$ à $130^{\circ} \mathrm{C}$, accroissement de $2^{\circ} / \mathrm{mn}$ et de $130^{\circ} \mathrm{C}$ à $200^{\circ} \mathrm{C}$ accroissement de $0,5^{\circ} / \mathrm{mn}$; gaz vecteur : azote ; pression d'entrée 1,7 bar ; débit : $15 \mathrm{ml} / \mathrm{mn}$.

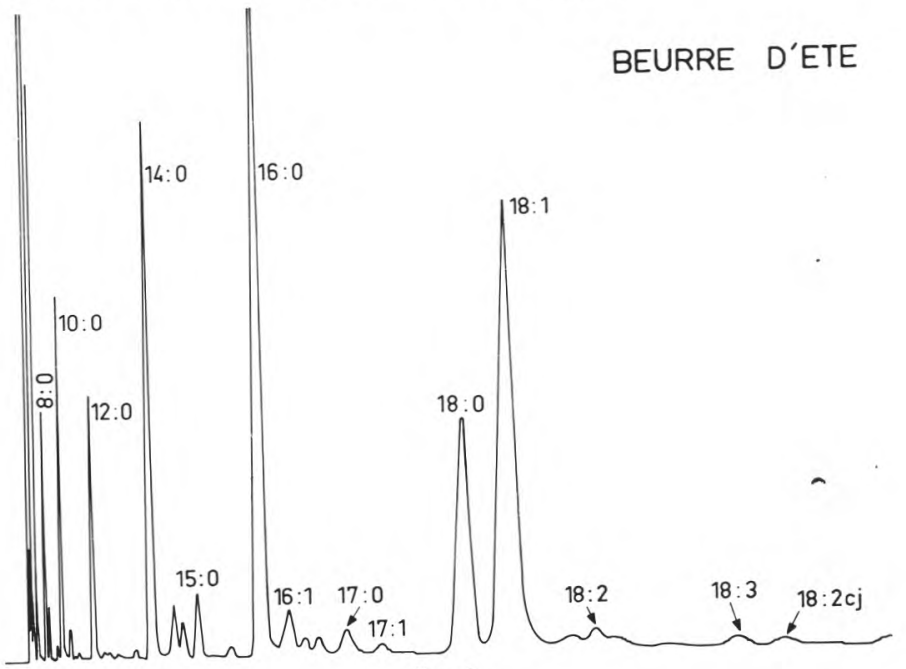

fig. 2

Analyse par chromatographie en phase gazeuse des acides gras du beurre de Retiers (beurre d'été).

Analyse isotherme à $200^{\circ} \mathrm{C}$. Autres conditions : Cf légende fig. 1 . 


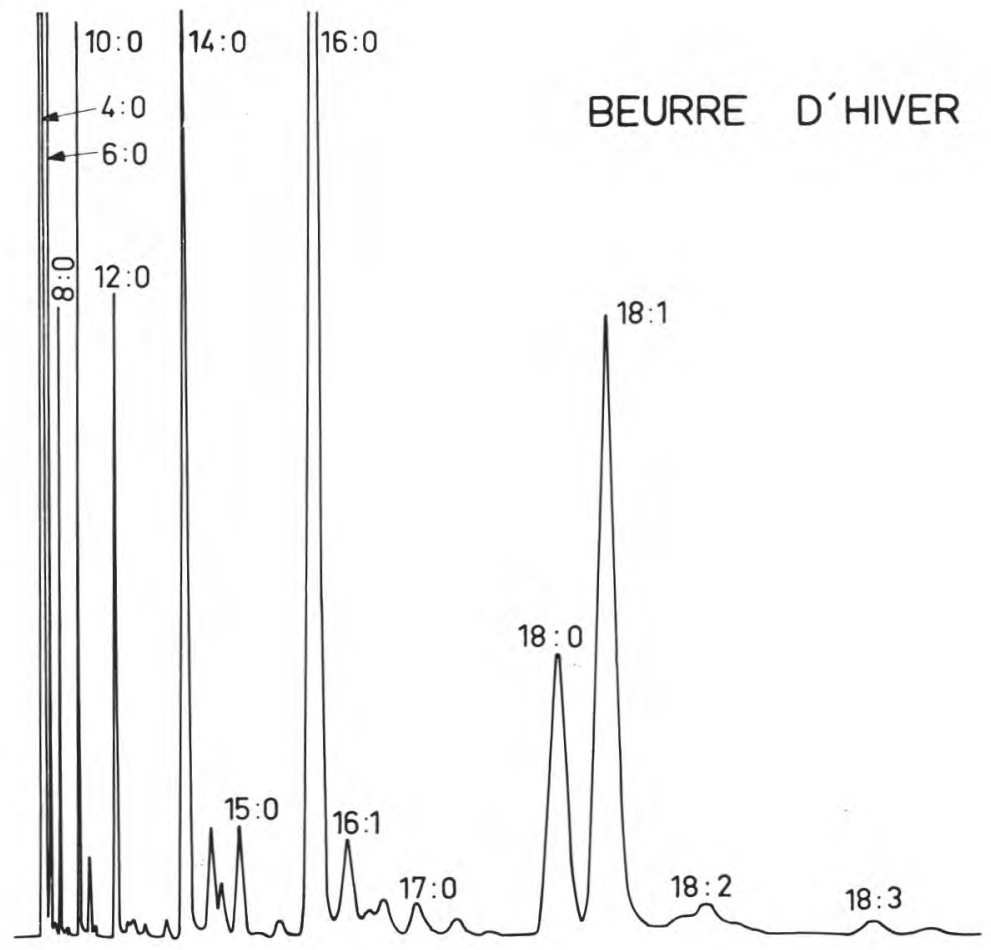

fig. 3

Analyse par chromatographie en phase gazeuse des acides gras du beurre de Retiers (beurre d'hiver).

Analyse isotherme à $200^{\circ} \mathrm{C}$. Autres conditions : Cf légende fig. 1 .

Tous les résultats, ou plus exactement les moyennes mensuelles des résultats, des analyses effectuées sur le beurre de Retiers au cours de l'année 1972 ont été rassemblés dans le tableau 1.

L'observation de ce tableau conduit à relever un certain nombre d'imperfections. Ainsi, dans les conditions isothermiques d'analyse, l'acide monoène à 14 atomes de carbone ne se sépare pas de l'acide ramifié (iso) comptant 15 atomes de carbone. Nous avons donc porté dans le tableau 1 la proportion globale de ces deux acides. A l'appellation 18:1 correspond, en fait, la somme du taux de l'acide oléique (cis-9-octadécènoïque) et du taux de l'acide vaccénique (trans 11 octadécènoïque.

L'appellation 18:2 correspond à l'évaluation globale du pic qui apparaît sur les figures 1, 2 et 3 après le pic de l'acide octadécènoïque. Or seule la partie médiane de ce pic correspond à l'acide linoléique 
TABLEAU 1

Moyennes mensuelles des proportions relatives des acides gras du beurre de Retiers (I-et-V.)

\begin{tabular}{|c|c|c|c|c|c|c|c|c|c|c|c|c|c|}
\hline & $\begin{array}{c}\text { Janv. } \\
1972\end{array}$ & $\begin{array}{c}\text { Févr. } \\
1972\end{array}$ & $\begin{array}{c}\text { Mars } \\
1972\end{array}$ & $\begin{array}{c}\text { Avril } \\
1972\end{array}$ & $\begin{array}{l}\text { Mai } \\
1972\end{array}$ & $\begin{array}{l}\text { Juin } \\
1972\end{array}$ & $\begin{array}{l}\text { Juil. } \\
1972\end{array}$ & $\begin{array}{l}\text { Août } \\
1972\end{array}$ & $\begin{array}{l}\text { Sept. } \\
1972\end{array}$ & $\begin{array}{l}\text { Oct. } \\
1972\end{array}$ & $\begin{array}{l}\text { Nov. } \\
1972\end{array}$ & $\begin{array}{l}\text { Déc. } \\
1972\end{array}$ & $\begin{array}{c}\text { Janv. } \\
1973\end{array}$ \\
\hline $4: 0$ & 3,60 & 4,01 & 3,70 & 3,90 & 3,50 & 3,15 & 3,40 & 3,20 & 3,20 & 3,15 & 3,15 & 3,75 & 3,85 \\
\hline $6: 0$ & 2,26 & 2,60 & 2,27 & 2,30 & 2,10 & 2,35 & 2,10 & 1,95 & 1,95 & 2,03 & 2,17 & 2,40 & 2,44 \\
\hline $8: 0$ & 1,36 & 1,47 & 1,40 & 1,50 & 1,45 & 1,35 & 1,30 & 1,15 & 1,20 & 1,30 & 1,35 & 1,46 & 1,53 \\
\hline $10: 0$ & 3,06 & 3,15 & 3,15 & 3,35 & 3,30 & 3,05 & 2,75 & 2,41 & 2,75 & 2,90 & 2,95 & 3,20 & 3,37 \\
\hline $10: 1$ & 0,28 & 0,29 & 0,27 & 0,30 & 0,30 & 0,25 & 0,27 & 0,25 & 0,30 & 0,31 & 0,32 & 0,34 & 0,34 \\
\hline $12: 0$ & 3,65 & 3,75 & 3,72 & 4,15 & 3,90 & 3,43 & 3,13 & 2,71 & 3,00 & 3,30 & 3,37 & 3,66 & 3,97 \\
\hline $12: 1$ & 0,16 & 0,10 & 0,11 & 0,14 & 0,12 & 0,12 & 0,11 & 0,10 & 0,15 & 0,15 & 0,14 & 0,13 & 0,14 \\
\hline il3 & 0,11 & 0,08 & 0,08 & 0,12 & 0,11 & 0,08 & 0,08 & 0,06 & 0,10 & 0,13 & 0,13 & 0,10 & 0,12 \\
\hline $13: 0$ & 0,09 & 0,08 & 0,08 & 0,10 & 0,10 & 0,09 & 0,09 & 0,08 & 0,10 & 0,11 & 0,10 & 0,10 & 0,10 \\
\hline i14 & 0,18 & 0,15 & 0,15 & 0,14 & 0,20 & 0,20 & 0,20 & 0,17 & 0,20 & 0,20 & 0,19 & 0,18 & 0,13 \\
\hline $14: 0$ & 11,50 & 11,65 & 11,20 & 10,90 & 10,90 & 10,50 & 10,25 & 9,50 & 9,75 & 10,60 & 10,80 & 11,20 & 11,90 \\
\hline $14: 1+\mathrm{i} 15$ & 1,29 & 1,28 & 1,15 & 1,20 & 1,35 & 1,40 & 1,40 & 1,30 & 1,45 & 1,50 & 1,55 & 1,55 & 1,57 \\
\hline ai15 & 0,59 & 0,50 & 0,49 & 0,66 & 0,75 & 0,85 & 0,80 & 0,70 & 0,75 & 0,75 & 0,72 & 0,66 & 0,61 \\
\hline $15: 0$ & 1,40 & 1,30 & 1,20 & 1,30 & 1,45 & 1,55 & 1,55 & 1,50 & 1,60 & 1,60 & 1,60 & 1,55 & 1,50 \\
\hline $15: 1$ & tr. & tr. & tr. & tr. & tr. & 0,10 & 0,10 & 0,07 & 0,15 & 0,10 & 0,11 & 0,09 & 0,06 \\
\hline i16 & 0,32 & 0,27 & 0,27 & 0,27 & 0,30 & 0,35 & 0,35 & 0,31 & 0,40 & 0,40 & 0,39 & 0,35 & 0,32 \\
\hline $16: 0$ & 30,50 & 30,90 & 28,15 & 24,35 & 24,10 & 23,60 & 24,20 & 24,10 & 24,00 & 25,70 & 27,10 & 28,10 & 30,00 \\
\hline $16: 1$ & 2,01 & 2,03 & 1,92 & 1,85 & 1,87 & 1,95 & 1,90 & 2,00 & 2,20 & 2,11 & 2,30 & 2,31 & 2,21 \\
\hline i17 & 0,41 & 0,26 & 0,45 & 0,55 & 0,73 & 0,65 & 0,60 & 0,60 & 0,70 & 0,60 & 0,60 & 0,60 & 0,51 \\
\hline ai17 & 0,82 & 0,30 & 0.80 & 0,86 & $0 ; 92$ & 0,90 & 0,80 & 0,73 & 0,90 & 0,90 & 1,00 & 1,05 & 0,90 \\
\hline $17: 0$ & 0,84 & 0,69 & 0,76 & 0,85 & 0,96 & 1,10 & 1,02 & 1,05 & 1,35 & 1,10 & 1,07 & 1,07 & 0,92 \\
\hline $17: 1$ & 0,39 & 0,39 & 0,33 & 0,40 & 0,46 & 0,46 & 0,43 & 0,45 & 0,60 & 0,50 & 0,55 & 0,57 & 0,48 \\
\hline i18 & 0,05 & 0,12 & 0,04 & 0,05 & 0,09 & 0,03 & 0,04 & 0,11 & 0,05 & 0,05 & 0,13 & 0,12 & 0,08 \\
\hline $18: 0$ & 9,85 & 9,55 & 10,40 & 11,25 & 11,95 & 12,70 & 12,90 & 13,10 & 12,30 & 11,30 & 10,40 & 9,90 & 9,22 \\
\hline $18: 1$ & 22,60 & 22,40 & 24,10 & 25,00 & 25,20 & 25,65 & 26,40 & 28,60 & 27,00 & 25,40 & 24,30 & 22,70 & 21,20 \\
\hline $18: 2$ & 1,65 & 1,52 & 2,03 & 2,05 & 1,60 & 1,80 & 1,60 & 1,55 & 1,95 & 2,03 & 1,97 & 1,66 & 1,52 \\
\hline- & 0,11 & 0,20 & 0,72 & 0,53 & 0,41 & 0,45 & 0,39 & 0,39 & 0,45 & 0,13 & 0,15 & 0,11 & 0,10 \\
\hline $18: 3$ & 0,63 & 0,65 & 0,74 & 0,90 & 0,75 & 0,92 & 0,96 & 0,88 & 0,90 & 0,85 & 0,84 & 0,72 & 0,62 \\
\hline $18: 2 \mathrm{cj}$ & 0,29 & 0,31 & 0,32 & 1,03 & 1,13 & 0,97 & 0,88 & 0,89 & 0,55 & 0,80 & 0,55 & 0,37 & 0,24 \\
\hline
\end{tabular}




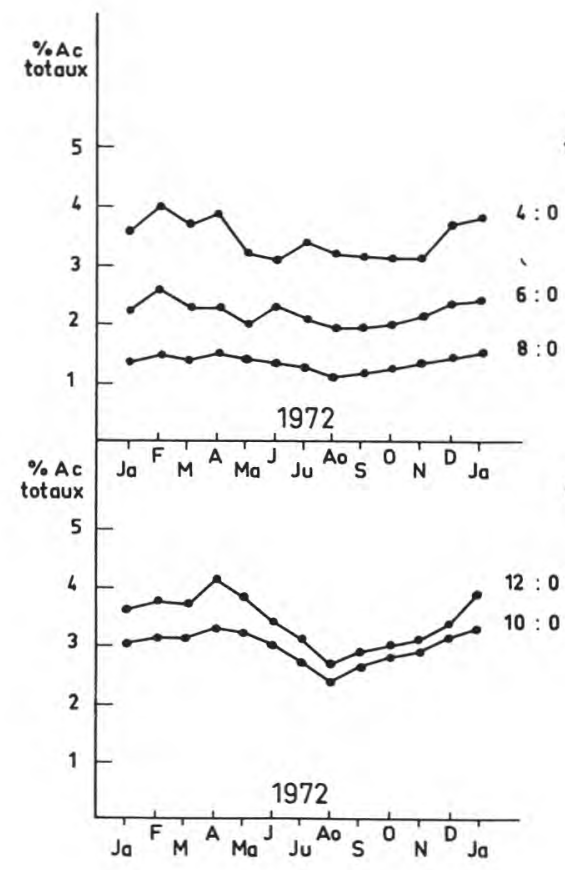

fig. 4

Variations saisonnières des proportions relatives des acides gras saturés à chaîne courte (4:0 à 12:0).

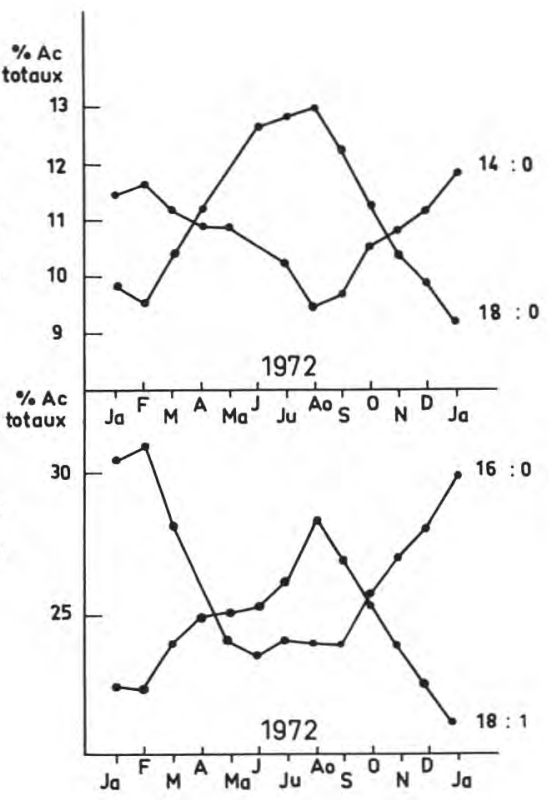

fig. 5

Variations saisonnières des proportions relatives des acides gras majeurs du beurre de Retiers (14:0, 16:0, 18:0, 18:1).

cis-9, cis-12-octadécadiènoïque, les pics adjacents correspondant sans doute à des isomères de l'acide linoléique. Il ne nous a pas été possible d'identifier le pic isolé qui précède l'acide linolénique. Par contre, la résolution des pics correspondant respectivement à l'acide linolénique et à l'acide diène conjugué a toujours été satisfaisante. Nous n'avons pas tenu compte des pics mineurs dont le temps de rétention était supérieur au temps de rétention de l'acide diène conjugué.

En ce qui concerne le taux de l'acide butyrique, dans la mesure où la réponse de l'intégrateur est linéaire quelle que soit la densité des impulsions, le taux obtenu correspond pratiquement au taux réel. En effet, d'une part, la technique d'estérification permet d'éviter toute perte d'acides volatils et, d'autre part, les coefficients de réponse des esters méthyliques des acides volatils (4:0, 6:0, 8:0) par rapport à l'ester méthylique de l'acide caprique (10:0) se sont révélés pratiquement égaux à 1 . 


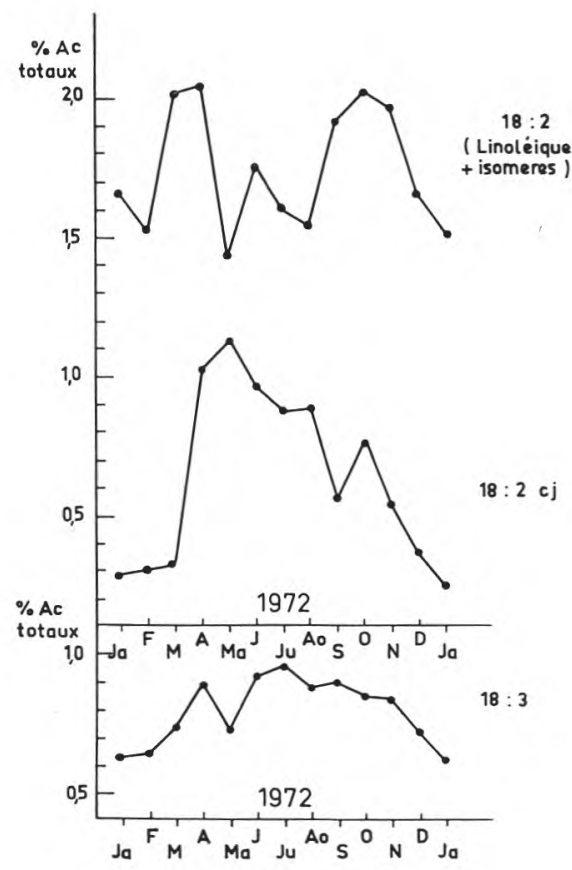

fig. 6

Variations saisonnières des proportions relatives des acides gras polyinsaturés du beurre de Retiers.

\section{2) Variations des proportions relatives des acides gras}

Les variations saisonnières des proportions relatives des acides gras saturés de 4 à 12 atomes de carbone sont représentées sur la figure 4 .

Les variations saisonnières des proportions relatives des acides majeurs (acide myristique, acide palmitique, acide stéarique et acide oléique, ou plus exactement oléique plus vaccénique) sont représentées sur la figure 5 .

Enfin, la figure 6 se rapporte aux variations saisonnières des proportions relatives des acides gras polyinsaturés.

L'observation de ces différentes figures permet de constater que les proportions relatives des acides saturés comptant 4 à 16 atomes de carbone évoluent parallèlement : le taux minimal se situe généralement vers la fin de l'été (en août, le plus souvent). A cette date les proportions relatives des acides stéarique et oléique 
atteignent un taux maximal, et suivent par ailleurs, tout au cours de l'année, une évolution inverse de celle suivie par les proportions relatives des acides saturés comptant 4 à 16 atomes de carbone. Ceci est illustré d'une manière particulièrement nette sur la figure 5 se rapportant à la variation des proportions relatives des acides palmitique et stéarique.

Dans l'ensemble, on observe une amplitude de variation de l'ordre de 20 à 25 p. 100 du taux moyen de chaque acide.

Si la proportion d'acide linoléique semble indépendante de la saison, la proportion d'acide diène conjugué à 18 atomes de carbone suit une évolution qui, - en première approximation - s'aligne sur l'évolution suivie par les proportions relatives des acides stéarique et oléique. Cependant la proportion d'acide diène conjugué présente deux maxima : l'un se situe en avril-mai, l'autre en octobrenovembre.

La proportion d'acide linolénique est plus élevée en période de pâturage qu'en période hivernale (janvier, février).

L'observation des figures 5 et 6 montre qu'en février-mars s'amorce un bouleversement de la composition des acides gras du beurre de Retiers. Les effets de ce bouleversement ne disparaissent pratiquement qu'en janvier de l'année suivante. La période « hivernale » est donc, à Retiers, extrêmement courte. Elle se réduit à 2 mois : janvier et février.

\section{3) Evolution des acides mineurs}

Les acides saturés comptant 15 et 17 atomes de carbone évoluent d'une manière assez voisine. La proportion minimale est observée en février. Elle s'accroît ensuite, jusqu'en juin et reste jusqu'en décembre. Les acides ramifiés à 15 et 17 atomes de carbone montrent une évolution assez semblable.

Les variations saisonnières des proportions respectives des acides palmitique et palmitoléique ne sont pas parallèles. La proportion d'acide palmitoléique, - comme d'ailleurs celle d'acide heptadécanoïque - est maximale en automne et diminue pendant l'hiver, période pendant laquelle la proportion d'acide palmitique est, au contraire maximale.

\section{4) Etude des rapports}

De nombreux auteurs, en Belgique, en Italie, en Espagne, ont évalué un certain nombre de rapports entre les proportions respectives des acides gras.

Nous avons porté dans le tableau 2 les valeurs de divers rapports calculés au cours de l'année. 
TABLEAU 2

Variations saisonnières des rapports établis à partir des proportions relatives des acides gras du beurre de Retiers

\begin{tabular}{|c|c|c|c|c|c|c|c|c|c|c|c|c|c|}
\hline & $\begin{array}{c}\text { Janv. } \\
1972\end{array}$ & $\begin{array}{l}\text { Févr. } \\
1972\end{array}$ & $\begin{array}{c}\text { Mars } \\
1972\end{array}$ & $\begin{array}{c}\text { Avril } \\
1972\end{array}$ & $\begin{array}{l}\text { Mai } \\
1972\end{array}$ & $\begin{array}{l}\text { Juin } \\
1972\end{array}$ & $\begin{array}{l}\text { Juil. } \\
1972\end{array}$ & $\begin{array}{l}\text { Août } \\
1972\end{array}$ & $\begin{array}{l}\text { Sept. } \\
1972\end{array}$ & $\begin{array}{l}\text { Oct. } \\
1972\end{array}$ & $\begin{array}{l}\text { Nov. } \\
1972\end{array}$ & $\begin{array}{l}\text { Déc. } \\
1972\end{array}$ & $\begin{array}{c}\text { Janv. } \\
1973\end{array}$ \\
\hline $4: 0 / 6: 0$ & 1,59 & 1,54 & 1,62 & 1,69 & 1,66 & 1,34 & 1,62 & 1,64 & 1,64 & 1,55 & 1,45 & 1,56 & 1,58 \\
\hline $\begin{array}{c}4: 0 \\
6: 0+8: 0\end{array}$ & 1,00 & 0,99 & 1,01 & 1,02 & 0,98 & 0,85 & 1,00 & 1,03 & 1,01 & 0,95 & 0,89 & 0,97 & 1,02 \\
\hline$\frac{4: 0+10: 0}{6: 0+8: 0}$ & 1,84 & 1,76 & 1,87 & 1,91 & 1,92 & 1,67 & 1,81 & 1,81 & 1,89 & 1,82 & 1,73 & 1,80 & 1,82 \\
\hline $6: 0 / 8: 0$ & 1,66 & 1,77 & 1,62 & 1,53 & 1,40 & 1,74 & 1,62 & 1,70 & 1,63 & 1,56 & 1,61 & 1,64 & 1,59 \\
\hline $10: 0 / 8: 0$ & 2,25 & 2,14 & 2,25 & 2,23 & 2,28 & 2,26 & 2,12 & 2,10 & 2,29 & 2,23 & 2,18 & 2,19 & 2,20 \\
\hline $12: 0 / 10: 0$ & 1,19 & 1,19 & 1,18 & 1,24 & 1,18 & 1,12 & 1,14 & 1,12 & 1,09 & 1,14 & 1,14 & 1,14 & 1,18 \\
\hline $14: 0 / 12: 0$ & 3,15 & 3,10 & 3,01 & 2,63 & 2,79 & 3,06 & 3,27 & 3,50 & 3,25 & 3,21 & 3,20 & 3,06 & 2,99 \\
\hline $14: 0 / 8: 0$ & 8,45 & 7,93 & 8,00 & 7,26 & 7,62 & 7,78 & 7,88 & 8,26 & 8,12 & 8,15 & 8,00 & 7,67 & 7,78 \\
\hline $16: 0 / 14: 0$ & 2,65 & 2,65 & 2,51 & 2,23 & 2,21 & 2,25 & 2,36 & 2,54 & 2,46 & 2,42 & 2,51 & 2,51 & 2,52 \\
\hline $16: 0 / 18: 0$ & 3,09 & 3,24 & 2,70 & 2,16 & 2,01 & 1,86 & 1,87 & 1,84 & 1,95 & 2,27 & 2,61 & 2,84 & 3,25 \\
\hline $18: 0 / 16: 0$ & 0,32 & 0,31 & 0,37 & 0,46 & 0,49 & 0,54 & 0,53 & 0,54 & 0,51 & 0,44 & 0,38 & 0,35 & 0,31 \\
\hline $18: 1 / 18: 0$ & 2,29 & 2,34 & 2,32 & 2,22 & 2,11 & 2,02 & 2,05 & 2,18 & 2,19 & 2,25 & 2,34 & 2,29 & 2,30 \\
\hline $18: 1 / 16: 0$ & 0,74 & 0,72 & 0,86 & 1,03 & 1,05 & 1,08 & 1,09 & 1,19 & 1,12 & 0,99 & 0,89 & 0,81 & 0,71 \\
\hline $18: 3 / 18: 2$ & 0,38 & 0,43 & 0,36 & 0,44 & 0,47 & 0,51 & 0,60 & 0,57 & 0,47 & 0,42 & 0,43 & 0,43 & 0,40 \\
\hline
\end{tabular}


Alors que le rapport acide palmitique / acide stéarique varie au cours de l'année presque du simple au double, les rapports acide palmitique/acide myristique ou acide laurique/acide caprique ou même acide butyrique / somme des acides caproïque et caprylique, montrent une remarquable constance.

\section{IV. - DISCUSSION}

\section{1) Etude comparée du beurre de Retiers et du beurre produit dans d'autres régions}

Nous n'avons pas observé, lors de notre étude, les brusques et profondes variations de composition qui se produisent dans les régions où les vaches laitières passent sans transition, d'un régime de stabulation permanente, avec une alimentation hivernale, à un régime de vie au pâturage comportant une alimentation à l'herbe exclusivement (Kuzdzal-Savoie et Kuzdzal, 1961, 1967 a).

Ainsi, à l'exception, toutefois, de la proportion d'acide palmitique qui s'abaisse nettement - de 31 à 24 p. 100 des acides totaux - de février à avril et de la proportion des acides diènes conjugués qui s'élève pendant la même période de 0,3 à 1,1 p. 100 (valeur maximale), les proportions de tous les autres acides s'élèvent ou s'abaissent graduellement.

Dans l'ensemble, l'amplitude de variation des proportions relatives des différents acides gras de beurre Retiers reste modérée. Elle représente environ les $2 / 3$ de l'amplitude des variations observées sur le beurre d'Echiré (Deux-Sèvres) (Kuzdzal-Savoie, 1967 b).

En vue de « situer " le beurre de Retiers dans l'ensemble des fabrications beurrières, nous avons rassemblé dans le tableau 3 les valeurs moyennes extrêmes des proportions relatives des acides gras majeurs de beurre fabriqué dans divers pays d'Europe. Ces moyennes ont été établies soit à partir des résultats obtenus sur les beurres fabriqués dans une région (nord-ouest de la Belgique : Guyot, 1965 ; province d'Emilie en Italie : Strocchi et al., 1967), soit à partir des résultats obtenus sur les beurres fabriqués dans un pays entier (Espagne : Garcia-Olmedo et al., 1971 ; Angleterre et Pays de Galles : Hall, 1970 ; Belgique : Huyghebaert, 1971 ; Pologne : Budlawski et al., 1969 ; Suisse : Hadorn et Zürcher, 1969, 1970). Quelques résultats concernant les Pays-Bas (Roos, 1963) et se rapportant aux acides gras à courte chaîne ont été incorporés dans le tableau 3. De plus, les mois pendant lesquels les proportions maximales ou minimales des différents acides gras sont obtenues, ont été indiqués. L'observation des résultats rassemblés dans le tableau 3 montre que l'amplitude des variations des proportions relatives des acides gras majeurs reste relativement faible dans les pays méditer- 
TABLEAU 3

Proportions relatives des acides gras majeurs du beurre. Limites de variation (observées dans différents pays d'Europe)

\begin{tabular}{|c|c|c|c|c|c|c|c|c|c|c|c|c|c|c|c|c|c|c|}
\hline & \multicolumn{2}{|c|}{$4: 0$} & \multicolumn{2}{|c|}{$6: 0$} & \multicolumn{2}{|c|}{$8: 0$} & \multicolumn{2}{|c|}{$10: 0$} & \multicolumn{2}{|c|}{$12: 0$} & \multicolumn{2}{|c|}{$14: 0$} & \multicolumn{2}{|c|}{$16: 0$} & \multicolumn{2}{|c|}{$18: 0$} & \multicolumn{2}{|c|}{$18: 1$} \\
\hline & Max. & Min. & Max. & Min. & Max. & Min. & Max. & Min. & Max. & Min. & Max. & Min. & Max. & Min. & Max. & Min. & Max. & Min. \\
\hline $\begin{array}{l}\text { Beurre de Retiers (Ille- } \\
\text { et-Vilaine), } 1972\end{array}$ & $\stackrel{4,01}{\mathrm{~F}}$ & $\stackrel{3,03}{\mathrm{~J}}$ & $\stackrel{2,60}{\mathrm{~F}}$ & $\begin{array}{l}1,94 \\
\text { Ao }\end{array}$ & $\begin{array}{c}1,48 \\
\mathrm{~A}\end{array}$ & $\begin{array}{l}1,14 \\
\text { Ao }\end{array}$ & $\begin{array}{c}3,22 \\
\mathrm{~A}\end{array}$ & $\begin{array}{l}2,41 \\
\text { Ao }\end{array}$ & $\begin{array}{c}4,12 \\
\mathrm{~A}\end{array}$ & $\begin{array}{c}2,71 \\
\text { Ao }\end{array}$ & $\stackrel{11,65}{\mathrm{~F}}$ & $\begin{array}{l}9,48 \\
\text { Ao }\end{array}$ & $\begin{array}{c}30,92 \\
\mathrm{~F}\end{array}$ & $\begin{array}{c}23,62 \\
S\end{array}$ & $\begin{array}{c}12,98 \\
\text { Ao }\end{array}$ & $\begin{array}{c}9,55 \\
\mathrm{~F}\end{array}$ & $\begin{array}{c}28,38 \\
\text { Ao }\end{array}$ & $\begin{array}{c}22,39 \\
F\end{array}$ \\
\hline $\begin{array}{l}\text { Angleterre et Pays de } \\
\text { Galles : Hall, } 1963\end{array}$ & $\begin{array}{l}3,9 \\
\text { M }\end{array}$ & $\stackrel{2,8}{\mathrm{~N}}$ & $\begin{array}{l}2,3 \\
\mathrm{Ju}\end{array}$ & $\begin{array}{l}1,5 \\
\mathrm{O}, \mathrm{N}\end{array}$ & $\begin{array}{l}1,4 \\
\mathrm{Ja}, \mathrm{Ju}\end{array}$ & $\begin{array}{l}1,0 \\
\text { Ao }\end{array}$ & $\begin{array}{l}3,2 \\
\mathrm{M}\end{array}$ & $\begin{array}{l}2,5 \\
\text { Ao }\end{array}$ & $\begin{array}{l}4,0 \\
\mathrm{M}\end{array}$ & $\begin{array}{l}3,1 \\
\text { Ao }\end{array}$ & $\mathrm{M}^{12,4}$ & $\begin{array}{l}10,4 \\
\text { Ao }\end{array}$ & $\begin{array}{c}29,3 \\
M\end{array}$ & $\begin{array}{c}23,8 \\
\text { Ao }\end{array}$ & $\begin{array}{l}13,2 \\
\text { Ao }\end{array}$ & $\begin{array}{l}9,2 \\
\mathrm{M}\end{array}$ & $\begin{array}{c}30,7 \\
\mathrm{~S}\end{array}$ & $\begin{array}{c}24,0 \\
M\end{array}$ \\
\hline $\begin{array}{l}\text { Belgique, Région I : } \\
\text { Guyot, } 1965\end{array}$ & - & - & $\begin{array}{l}3,0 \\
M\end{array}$ & $\begin{array}{l}2,4 \\
\text { Ao }\end{array}$ & $\begin{array}{l}1,8 \\
M\end{array}$ & $\begin{array}{l}1,5 \\
\text { Ao }\end{array}$ & $\begin{array}{l}3,4 \\
\mathrm{Ma}\end{array}$ & $\begin{array}{l}2,7 \\
\text { Ao }\end{array}$ & $\begin{array}{l}3,6 \\
\mathrm{Ja}\end{array}$ & $\begin{array}{l}2,8 \\
\mathrm{Ju}\end{array}$ & $\begin{array}{c}12,0 \\
\mathrm{Ja}\end{array}$ & $\stackrel{9,7}{S}$ & $\begin{array}{c}31,4 \\
F\end{array}$ & $\begin{array}{l}22,4 \\
\mathrm{Ma}, \mathrm{J}\end{array}$ & $\begin{array}{l}12,6 \\
\text { Ao }\end{array}$ & $\begin{array}{l}8,3 \\
\mathrm{Ja}\end{array}$ & $\begin{array}{c}29,9 \\
0\end{array}$ & $\begin{array}{c}22,5 \\
\mathrm{~J}\end{array}$ \\
\hline $\begin{array}{l}\text { Belgique : Huygebeart, } \\
\text { Hendrickx, } 1971\end{array}$ & $\begin{array}{l}4,0 \\
\mathrm{M}\end{array}$ & $\begin{array}{l}3,6 \\
\text { AoàD }\end{array}$ & $\stackrel{2,2}{A, S, N}$ & $\stackrel{2,0}{\mathrm{D}}$ & $\stackrel{1,3}{J}$ & $\begin{array}{c}1,1 \\
\text { D,Ju, } \\
\text { A,O }\end{array}$ & $\stackrel{3,2}{\mathrm{~J}}$ & $\begin{array}{c}2,2 \\
\mathrm{Ao}, \mathrm{S}, \\
\mathrm{M}\end{array}$ & $\begin{array}{c}3,8 \\
\mathrm{~J}\end{array}$ & $\begin{array}{l}2,7 \\
\text { Ao }\end{array}$ & $\begin{array}{c}11,3 \\
\mathrm{Ja}\end{array}$ & $\begin{array}{c}9,6 \\
\text { Ju, O }\end{array}$ & $\stackrel{28,1}{A}$ & $\underset{\mathrm{J}}{21,9}$ & $\begin{array}{c}13,2 \\
\mathrm{Ju}\end{array}$ & $\begin{array}{c}9,6 \\
0\end{array}$ & $\begin{array}{c}31,5 \\
0\end{array}$ & $\begin{array}{c}26,6 \\
\mathrm{Ja}\end{array}$ \\
\hline $\begin{array}{l}\text { Italie (Emilia) : Stroc- } \\
\text { chi et al., } 1967\end{array}$ & $\stackrel{4,4}{J}$ & $\stackrel{2,6}{F}$ & $\begin{array}{l}2,5 \\
\mathrm{Ma}\end{array}$ & $\stackrel{1,6}{F}$ & $\begin{array}{c}2,0 \\
\mathrm{Ma}, \mathrm{J}\end{array}$ & $\begin{array}{l}1,1 \\
\mathrm{O}, \mathrm{N}\end{array}$ & $\begin{array}{l}3,3 \\
\mathrm{Ma}\end{array}$ & $\begin{array}{r}2,8 \\
\mathrm{~N}, \mathrm{D}\end{array}$ & $\begin{array}{l}3,8 \\
\mathrm{Ja}, \mathrm{S}\end{array}$ & $\stackrel{3,2}{\mathrm{~J}}$ & $\begin{array}{c}12,4 \\
\mathrm{Ja}\end{array}$ & $\begin{array}{c}10,2 \\
S\end{array}$ & $\stackrel{33,1}{\mathrm{~F}}$ & $\underset{\mathrm{J}}{27,2}$ & $\begin{array}{c}10,4 \\
\mathrm{Ju}\end{array}$ & $\begin{array}{c}9,2 \\
\mathrm{~F}, \mathrm{Ma}, \\
\text { Ao,N }\end{array}$ & $\begin{array}{c}23,9 \\
\text { Ju }\end{array}$ & $\begin{array}{c}21,3 \\
\mathrm{~N}\end{array}$ \\
\hline $\begin{array}{l}\text { Espagne : Olmedo et } \\
\text { al., } 1971\end{array}$ & $\begin{array}{c}4,53 \\
\mathrm{~A}\end{array}$ & $\stackrel{3,80}{\mathrm{~J}}$ & $\begin{array}{c}2,84 \\
\mathrm{M}\end{array}$ & $\begin{array}{c}2,56 \\
\mathrm{~N}\end{array}$ & $\begin{array}{c}1,58 \\
\mathrm{M}\end{array}$ & $\stackrel{1,41}{\mathrm{~N}}$ & $\begin{array}{c}3,33 \\
F\end{array}$ & $\begin{array}{c}2,79 \\
\mathrm{~N}\end{array}$ & $\stackrel{3,74}{\mathrm{~F}}$ & $\begin{array}{c}3,12 \\
\text { Ao }\end{array}$ & $\stackrel{10,76}{F}$ & $\begin{array}{l}9,79 \\
\text { Ao }\end{array}$ & $\stackrel{25,58}{F}$ & $\begin{array}{c}22,93 \\
\text { Ao }\end{array}$ & $\begin{array}{c}12,66 \\
\mathrm{Ju}\end{array}$ & $\stackrel{10,77}{F}$ & $\begin{array}{c}24,27 \\
\text { Ao }\end{array}$ & $\begin{array}{c}21,4 \\
F\end{array}$ \\
\hline $\begin{array}{l}\text { Suisse : Hadorn, Zür- } \\
\text { cher, } 1970\end{array}$ & $\stackrel{4,13}{J}$ & $\stackrel{3,46}{\mathrm{~N}}$ & $\stackrel{2,43}{F}$ & $\begin{array}{l}1,75 \\
\mathrm{~S}\end{array}$ & $\stackrel{1,4}{A}$ & $\stackrel{0,8}{\mathrm{~N}}$ & $\stackrel{3,1}{\mathrm{~A}}$ & $\stackrel{1,8}{N}$ & $\stackrel{3,6}{\mathrm{D}}$ & $\stackrel{2,2}{\mathbf{J}}$ & $\begin{array}{c}11,6 \\
\mathrm{D}\end{array}$ & $\stackrel{8,6}{S}$ & $\begin{array}{c}30,6 \\
\mathrm{D}\end{array}$ & $\begin{array}{c}21,3 \\
\text { Ao }\end{array}$ & $\begin{array}{c}11,5 \\
\mathrm{~J}\end{array}$ & $\begin{array}{c}7,7 \\
\mathrm{D}\end{array}$ & $\begin{array}{c}30,5 \\
\mathrm{~S}\end{array}$ & $\begin{array}{c}21,1 \\
\mathrm{D}\end{array}$ \\
\hline $\begin{array}{l}\text { Pologne : Budslawski } \\
\text { et al., } 1969\end{array}$ & $\begin{array}{c}5,37 \\
\mathrm{Ja}\end{array}$ & $\begin{array}{l}2,03 \\
\mathrm{Ma}\end{array}$ & $\begin{array}{c}3,56 \\
\mathrm{Ja}\end{array}$ & $\begin{array}{l}1,65 \\
\mathrm{Ma}\end{array}$ & $\begin{array}{c}2,14 \\
\mathrm{Ja}\end{array}$ & $\begin{array}{l}1,07 \\
\mathrm{Ma}\end{array}$ & $\begin{array}{c}3,95 \\
\mathrm{Ja}\end{array}$ & $\begin{array}{l}2,16 \\
\mathrm{Ma}\end{array}$ & $\begin{array}{c}4,24 \\
\mathrm{Ja}\end{array}$ & $\begin{array}{l}2,45 \\
\mathrm{Ma}\end{array}$ & $\begin{array}{c}13,01 \\
\mathrm{Ja}\end{array}$ & $\begin{array}{l}8,35 \\
\mathrm{Ma}\end{array}$ & $\begin{array}{c}34,61 \\
\mathrm{D}\end{array}$ & $\begin{array}{c}23,31 \\
\mathrm{~S}\end{array}$ & $\begin{array}{c}11,84 \\
\text { J }\end{array}$ & $\begin{array}{c}5,02 \\
\mathrm{Ja}\end{array}$ & $\begin{array}{l}29,73 \\
\mathrm{Ma}\end{array}$ & $\begin{array}{c}15,18 \\
\mathrm{Ja}\end{array}$ \\
\hline Pays-Bas : Roos, 1963 & $\begin{array}{l}3,3 \\
\mathrm{M}\end{array}$ & $\begin{array}{c}2,8 \\
\mathrm{O}\end{array}$ & & & & & & & & & & & & & & & & \\
\hline
\end{tabular}

Ja : Janvier ; F : Février ; M : Mars ; A : Avril ; Ma : Mai ; J : Juin ; Ju : Juillet ; Ao : Août ; S : Septembre ; O : Octobre ; $\mathrm{N}$ : Novembre ; D : Décembre. 
ranéens (Espagne et Italie) et est par contre très forte en Pologne, pays au climat continental, et à un moindre degré en Belgique. Elle reste modérée en Angleterre (et Pays de Galles) comme d'ailleurs à Retiers (climat océanique relativement doux).

Les variations de composition des beurres offrent un certain parallélisme : en janvier, février et mars, les proportions des acides stéarique et oléique sont partout minimales et les proportions des acides myristique et palmitique sont alors maximales. Les proportions maximales des acides oléique et stéarique sont observées au printemps (Pologne), en été (Retiers, Angleterre, Espagne, Italie) ou en automne (Belgique). A Retiers, comme parfois ailleurs, l'accroissement de taux d'acide stéarique précède l'accroissement du taux d'acide oléique.

\section{2) Origine des variations observées}

Les acides gras du lait ont une double origine. Les acides de 4 à 16 atomes de carbone sont synthétisés dans la glande mammaire à partir de l'acide acétique présent dans le sang. L'acide acétique résulte lui-même des fermentations dont le rumen est le siège ; en outre de petites quantités d'acide propionique, d'acide isobutyrique, d'acide isovalérique ou d'acide 2-méthyl-propionique se forment également lors de ces fermentations, et, par la même voie biosynthétique (additions successives de maillons à 2 atomes de carbone), peuvent conduire aux acides gras à nombre impair d'atomes de carbone et aux acides ramifiés (iso et antéiso).

La glande mammaire prélève dans le sang les acides à longue chaîne (palmitique, stéarique, oléique) qui s'y trouvent. Ces acides à longue chaîne proviennent de l'alimentation ou des réserves, ou sont formés par biosynthèse dans divers organes (dans le foie par exemple). Les acides gras alimentaires saturés peuvent dans une certaine mesure passer dans le sang puis dans le lait sans modification. Mais les acides polyinsaturés subissent une hydrogénation au niveau du rumen sous l'action des micro-organismes (bactéries et protozoaires) et les acides linolénique et linoléique sont ainsi normalement transformés en acide oléique et stéarique. Cette hydrogénation s'accompagne de la formation d'acides trans (acide vaccénique) et d'acides diènes conjugués à 18 atomes de carbone. Par ailleurs, une désaturase agissant sous contrôle hormonal et présente dans la glande mammaire permet une transformation de l'acide stéarique en acide oléique.

Compte tenu de ce qui précède, l'influence de l'alimentation des vaches laitières sur la composition des acides gras du beurre apparaît primordiale. En effet, l'apport d'aliments hydrocarbonés favorise la formation d'acides saturés jusqu'à 16 atomes de carbore et l'apport d'herbe plus spécialement d'herbe jeune de printemps ou d'automne (regains), riche en acides polyinsaturés (4 p. 100 
environ de l'extrait sec) favorise la formation d'acide stéarique et d'acide oléique.

Dans la région de Retiers, les vaches laitières restent au pâturage pratiquement toute l'année et ne rentrent à l'étable qu'à l'heure de la traite. L'herbe ou les fourrages frais constituent donc l'essentiel de leur alimentation à l'exception toutefois de la période d'hiver pendant laquelle elles reçoivent une alimentation d'appoint constituée par de l'ensilage de maïs auquel s'ajoute une quantité variable de foin. Les variations de composition des acides gras du beurre de Retiers s'expliquent donc aisément par l'influence primordiale du facteur alimentaire. Toutefois, l'influence de l'alimentation n'est pas seule en cause pour expliquer les variations saisonnières des proportions relatives des acides gras du beurre.

Une influence propre du stade de lactation peut s'ajouter à l'influence alimentaire. Dans la région de Retiers on pratique de plus en plus les vêlages d'automne, mais les vêlages de printemps existent encore. Il a été montré par divers auteurs (Kuzdzal, 1956 ; Decaen et Adda, 1966, entre autres) qu'au cours des 2 premiers mois de lactation, la sécrétion des acides saturés de 4 à 14 atomes de carbone est particulièrement élevée. Ceci peut entraîner ou expliquer un ralentissement de la diminution des proportions des acides saturés de 4 à 10 atomes de carbone au printemps et un accroissement de ces proportions à l'automne, période pendant laquelle en outre les vaches laitières commencent à recevoir l'aliment d'appoint.

Il convient de ne pas omettre le rôle propre joué par la flore du rumen. Indépendamment de son action hydrogénante, celle-ci constitue une source propre de "lipides alimentaires ". Keeney et al. (1962) ont en effet montré que plus de la moitié des acides ramifiés à 15 et 17 atomes de carbone du lait provenaient directement des lipides du protoplasme des bactéries du rumen, toujours riche en acides ramifiés à longue chaîne.

Si les variations des proportions d'acide linoléique et d'acide diène conjugué dans le beurre de Retiers montrent un certain parallélisme avec les variations atteignant les acides stéarique ou oléique, la variation propre de la proportion d'acide linoléique ne s'aligne sur aucune autre variation. L'étude poursuivie sur le beurre de Retiers confirme certaines études antérieures (Kuzdzal-Savoie et Kuzdzal, 1961 ; Guyot, 1965 ; Hall, 1970). La proportion d'acide C18:2 portée dans le tableau 1 correspond à la somme de l'acide linoléique vrai et des deux acides adjacents. Cependant il nous est apparu que la surface relative du pic central correspondant à l'acide linoléique vrai était plus forte en hiver qu'en période estivale.

Les variations des proportions des acides monoènes mineurs s'expliquent mal par une désaturation uniforme s'exerçant sur l'acide saturé correspondant; les proportions maximales et minimales des 
acides monoènes mineurs d'une part et des acides saturés majeurs d'autre part apparaissent en effet à des périodes différentes.

\section{3) Mise en évidence des relations entre les acides gras}

De nombreux auteurs ont établi la variation au cours de l'année d'une série de rapports calculés à partir des proportions connues des différents acides gras du beurre. Si aucun de ces rapports ne restent constants au cours de l'année, certains d'entre eux montrent une relative stabilité. Il en est ainsi, par exemple, du rapport 12:0/10:0 qui a souvent été proposé comme critère permettant de contrôler la pureté du beurre (Wolff, 1960 ; Boniforti, 1962 ; Francesco et Avancini, 1961 ; Guyot, 1968 a et b, entre autres).

Nous avons rassemblé dans le tableau 4 les valeurs extrêmes de divers rapports calculés par les auteurs à partir des résultats de leurs propres analyses. Strocchi et al. (1967) et Chioffi et Magon (1964) sont cités pour l'Italie, Kufferath (1968) et Huyghebaert et Hendrickx (1971) pour la Belgique, Janoschek et Metin (1968) pour l'Allemagne, Olmedo et Gastanaduy (1971) pour l'Espagne. Les rapports calculés pour le beurre de Retiers sont incorporés dans le tableau 4.

Nous avons volontairement éliminé de ce tableau le rapport 18:3/18:2. En effet, la phase stationnaire de succinate de diéthylène glycol la plus généralement utilisée ne permet pas de séparer le pic de l'acide linolénique du pic de l'acide diène conjugué, ce qui fausse tout calcul.

Nous n'avons pas retenu pour la présentation du tableau 4 les valeurs établies par Guyot et Piraux (1965). En effet, de nombreux résultats obtenus par ces auteurs au cours des années 1963, 1964 et 1965 proviennent d'analyses effectuées sur des laits individuels produits parfois par des vaches laitières en fin de lactation ou soumises à un régime alimentaire particulier, comportant par exemple la distribution de tourteaux de coprah. Ceci a pour effet de modifier notablement la valeur du rapport 12:0/10:0 (Kuzdzal et Paquot, 1962).

Les rapports calculés à partir des résultats obtenus sur le beurre de Retiers se situent pratiquement à l'intérieur des écarts proposés par les auteurs, pour tous les rapports à l'exception toutefois du rapport 4:0/6:0 qui est parfois inférieur à la limite proposée par Huyghebaert et Hendrickx.

Les valeurs du rapport 12:0/10:0 concernant le beurre de Retiers s'alignent également sur les résultats des différents auteurs en particulier sur ceux indiqués par Chioffi et Magon (1964).

Dans l'ensemble, l'écart observé entre les valeurs extrêmes des différents rapports calculés pour le beurre de Retiers est sensiblement plus étroit que les écarts indiqués par les auteurs.

Roos (1964) cependant, a présenté des objections à l'emploi des rapports des proportions d'acides gras en vue de contrôler la pureté 
TABLEAU 4

Etude comparée des rapports établis par divers auteurs à partir des proportions relatives des acides gras du beurre

\begin{tabular}{|c|c|c|c|c|c|c|c|}
\hline $\begin{array}{l}\text { Rapport entre } \\
\text { acides gras }\end{array}$ & $\begin{array}{l}\text { Beurre de } \\
\text { Retiers } \\
1972\end{array}$ & $\begin{array}{c}\text { Janosckek } \\
\text { et Metin } \\
1968\end{array}$ & $\begin{array}{l}\text { Strocchi } \\
\text { et al. } \\
1967\end{array}$ & $\begin{array}{l}\text { Chioffi } \\
\text { et Magon } \\
1964\end{array}$ & $\begin{array}{l}\text { Olmedo } \\
\text { et al. } \\
1971\end{array}$ & $\begin{array}{c}\text { Kufferath } \\
1968\end{array}$ & $\begin{array}{c}\text { Huyghebaert } \\
\text { et Hendrickx } \\
1971\end{array}$ \\
\hline $4: 0 / 6: 0$ & $1,34-1,69$ & - & - & - & - & - & $1,52-2,11$ \\
\hline$\frac{4: 0}{6: 0+8: 0}$ & $0,85-1,03$ & - & $0,76-1,25$ & $0,83-1,32$ & $0,74-1,33$ & $1,0-1,4$ & $0,72-1,16$ \\
\hline$\frac{4: 0+10: 0}{6: 0+8: 0}$ & $1,66-1,91$ & - & - & - & $1,46-2,21$ & $1,8-2,1$ & - \\
\hline $6: 0 / 8: 0$ & $1,40-1,77$ & $1,20-1,87$ & - & $1,33-2,44$ & $1,42-2,22$ & - & $1,42-2,22$ \\
\hline $10: 0 / 8: 0$ & $2,10-2,29$ & - & - & $1,67-2,30$ & $1,71-2,49$ & $1,8-2,3$ & $1,58-2,83$ \\
\hline $12: 0 / 10: 0$ & $1,09-1,24$ & $1,11-1,53$ & $0,92-1,38$ & $1,04-1,17$ & $1,00-1,20$ & $1,0-1,26$ & $1,06-1,36$ \\
\hline $14: 0 / 12: 0$ & $2,63-3,50$ & $2,78-3,65$ & $2,66-3,70$ & $3,04-3,81$ & $2,32-3,67$ & $2,7-3,7$ & $2,20-4,06$ \\
\hline $14: 0 / 8: 0$ & $7,26-8,45$ & - & - & - & $5,71-8,42$ & $6,7-9,1$ & - \\
\hline $16: 0 / 14: 0$ & $2,21-2,65$ & - & - & $2,16-2,70$ & $1,93-2,94$ & 3,0 env. & $1,87-2,95$ \\
\hline $18: 0 / 16: 0$ & $0,31-0,54$ & - & - & $0,21-0,52$ & - & - & $0,25-0,76$ \\
\hline $18: 1 / 16: 0$ & $0,71-1,19$ & - & - & - & - & - & $0,75-1,55$ \\
\hline $18: 1 / 18: 0$ & $2,02-2,34$ & - & $2,10-2,80$ & $2,05-3,42$ & $1,57-2,52$ & $2,2-3,1$ & $1,85-3,06$ \\
\hline
\end{tabular}


du beurre et a proposé une méthode qui utilise la combinaison linéaire de deux proportions d'acides gras au lieu du quotient.

A l'heure actuelle, de toute façon, la seule analyse des acides gras du beurre ne suffit plus à garantir la pureté de celui-ci. Par contre, l'évaluation des rapports tels qu'ils sont indiqués dans le tableau 4 permet à toute entreprise beurrière de connaître à tout moment les caractéristiques chimiques et souvent physico-chimiques du beurre qu'elle fabrique et, donc, d'être à même de répondre à certaines exigences nouvelles qui apparaissent dans les échanges commerciaux.

\section{4) Identification des acides gras mineurs}

Au cours de cette étude, nous avons régulièrement observé l'apparition sur les chromatogrammes de quelques pics mineurs pour lesquels nous n'avons pas été en mesure de donner une appellation certaine.

Il s'agit entre autres des deux pics adjacents au pic de l'acide linoléique (cis-9-cis-12-octadécadiènoïque), du pic qui précède le pic de l'acide linolénique, d'un pic adjacent à l'acide ramifié à 18 atomes de carbone et des pics postérieurs au pic de l'acide arachidique.

L'identification de ces pics mineurs déborde du cadre du présent travail mais une étude ultérieure pourrait y être consacrée. Les travaux récents de Strocchi et Holman (1971) ouvrent la voie à une telle recherche.

\section{R és u m é}

De janvier 1972 à janvier 1973, chaque semaine, un échantillon du beurre fabriqué dans la région de Retiers (Ille-et-Vilaine) a été analysé.

Les proportions relatives des acides gras ont été déterminées par chromatographie en phase gazeuse.

Les amplitudes de variation des proportions de chaque acide gras ont été établies. Ainsi, la proportion de l'acide palmitique a varié de 23,6 (en p. 100 des acides gras totaux) en juin, à 30,9 en février. La proportion de l'acide oléique a varié de 21,2 en décembre à 28,6 en août.

L'amplitude de variation de quelques rapports calculés à partir des proportions des divers acides gas a également été étudiée. Ainsi, le rapport entre la proportion d'acide laurique et la proportion d'acide caprique $(\mathrm{C} 12 / \mathrm{C} 10)$ a varié de 1,09 en septembre à 1,24 en avril.

Les résultats obtenus sur le beurre de Retiers ont été comparés aux résultats obtenus par différents auteurs sur des beurres fabriqués dans divers pays d'Europe. 
L'amplitude des variations saisonnières de composition du beurre de Retiers est relativement modérée et comparable à l'amplitude des variations observées sur les beurres fabriqués dans des pays ou régions soumis à un climat de nature océanique.

L'étude rapportée présente un intérêt sur le plan commercial elle permet, par exemple, de garantir le respect de certaines exigences nouvelles de composition portées parfois sur les cahiers des charges.

Les corrections de composition et, donc bien souvent de texture du beurre, obtenues par réincorporation de crèmes d'été dans des crèmes d'hiver pourront être désormais plus aisément suivies et dominées.

\section{Zusammenfassung}

\section{JAHRESZEITLICHE SCHWANKUNGEN DER RELATIVEN ANTEILEN AN FETTSAUREN EINER BUTTER HERGESTELLT IN RETIERS}

(ILLE-ET-VILAINE)

Von Januar 1972 bis Januar 1973 wurde jede Woche eine in dem Gebiete von Retiers (Ille-et-Vilaine) hergestellte Butterprobe untersucht. Die relative Anteilen der Fettsäuren wurden gaschromatographisch bestimmt.

Die Schwankungsbreiten der Anteilen jeder Fettsäure wurden festgestellt. So hat der Anteil an Palmitinsäure von 23,6 p. 100 (in p. 100 der Gesamt Fettsäuren) in Juni bis 30,9 p. 100 in Februar geschwankt. Der Anteil an Olsäure hat von 21,2 p. 100 in Dezember bis 28,6 p. 100 in August geschwankt.

Die erhaltenen Ergebnisse auf der Butter von Retiers wurden mit dem Ergebnissen von verschiedenen Autoren auf der hergestellten Butter von mehreren Ländern Europas, vergliechen.

Die Jahreszeitliche Schwankungsbreite der Zuzammensetzung der Butter von Retiers ist relativ mittelmässig und vergleichbar mit der auf Butter beobachtete Schwankungsbreiten, die in Ländern oder Gebieten unter der Wirkung eines Ozeanischen Klimas hergestellt sind.

Die berichteten Untersuchungen sind auf kaufmännischen Gebiet von Vorteil. Sie erlauben z.B., die Beachtung neuer Forderungen der Zusammensetzung, die manchmal auf Lieferungsbedingungen eingetragen sind, zu sichern.

Die verbesserten Zusammensetzungen der Butter und folglich oft Gefügeverbesserungen, z.B. durch Beimischung von Sommerrahm in Winterrahm, werden nun leichter befolgt und beherrscht. 


\section{S u m m a r y \\ SEASONAL VARIATIONS IN THE FATTY ACID COMPOSITION OF BUTTER MADE AT RETIERS (ILLE-ET-VILAINE)}

From January 1972 to January 1973, a sample of butter made in the region of Retiers (Ille-et-Vilaine) has been analysed each week.

The relative proportions of fatty acids were determined by means of gas chromatography.

The magnitudes of variation in the proportions of each fatty acid were established. Thus, the proportion of palmitic acid varied from 23.6 (in percent of total fatty acids) in June to 30.9 in February. The proportion of oleic acid varied from 21.2 in December to 28.6 in August.

The magnitude of variation of some ratios calculated from the proportions of the different fatty acids was also studied. The ratio between the proportion of lauric acid and capric acid $(\mathrm{C} 12 / \mathrm{C} 10)$ varied from 1.09 in September to 1.24 in April.

The results obtained with the butter from Retiers were compared with those recorded by different authors and concerning butter made in various European countries.

The magnitude of the seasonal variations in the composition of butter from Retiers was relatively moderate and comparable to the data recorded with butter made in countries or regions subjected to a climate of the oceanic type.

This study is interesting from a commercial point of view, it allows for instance to meet some of the recent requirements concerning butter composition sometimes indicated on the statement of works.

Corrections of the composition and consequently of the texture of the butter obtained by reincorporation of summer cream into winter cream can therefore be more easily followed and controlled.

Reçu pour publication le 18 janvier 1974.

\section{Références bibliographiques}

Bejambes (M.), Savoie (S.) (1954). - Etude comparée des variations saisonnières de I'indice d'iode des beurres, en Normandie et en Alsace. Chimie et Industrie, $71,501-506$.

BONIFORTI (L.) (1962). - Etude par chromatographie en phase gazeuse des acides gras du beurre fabriqué en Italie et dans d'autres pays. Application à la recherche des falsifications dans le beurre commercial. Ann. Fals. Exp. Chim., $55,255-263$.

BuDSLAWSKI (J.), JAWORSKA (H.), JAWORSKI (J.), TOMASIK (M.) (1969). - Sklad Chemiczny tluszczu mlekowego. Zeszyt y Naukowe W. Szk. Rol., Olsztyn, $25,364-398$. 
Chioffi (V.), MAGoN (G.) (1964). - Rapporti significativi ricavati dell'analisi gascromatografica di burri reperibili in commercio. Riv. Ital. Sost. Grasse, $41,243-254$.

Decaen (C.), Adda (J.) (1966). - Evolution de la sécrétion des acides gras des glycérides du lait de vache au cours de la lactation. XVII Congr. Int. Lait., Munich, A:1, 161-171.

Dreassi (M.), GUARINIERI (E.), VECchIONI (R.) (1970). - Il latte prodotto nell'Agro Romano: Caratteristiche e variabilità. Nota II : Tenore in grasso. Caratteristiche estudio gascromatografico. Boll. Laboratiri Chimici provinciali, $21,21-33$.

de Francesco (F.), Avancini (D.) (1961). - La sofisticazione del burro con grassi transesterificati. Il Latte, 35, 809-817.

de Francesco (F.), Avancini (D.), Maglitto (C.), Gandini (C.) (1961). - Cromatografia quantitativa in F.V. Nota III : Composizione degli acidi del grasso del latte di vacca. Rev. Ital. Sost. Grasse, 38, 307-314.

Guyot (A.L.) (1965). - Composition de la matière grasse des beurres belges et recherche des falsifications par la chromatographie en phase gazeuse. J. de Pharmacie de Belgique, 367-396.

Guyot (A. L.) (1968 a). - Détermination des falsifications du beurre par certaines graisses transestérifiées grâce à l'analyse des acides gras et des stéroïdes. Bull. Rech. Agron. Gembloux, 3, 128-157.

Guyot (A. L.) (1968 b). - Détermination des falsifications du beurre par certains saindoux industriels grâce à l'analyse des acides gras et des stéroïdes. Bull. Rech. Agron. Gembloux, 3, 471-502.

Guyot (A. L ), Piraux (E. F.) (1964). - Etude par la chromatographie en phase gazeuse de la matière grasse des beurres de laiteries belges. Bull. Inst. Agron. et Stat. Rech Gembloux, 32, 190-221.

Guyot (A. L.), Piraux (E.F.) (1965). - Etude par la chromatographie en phase gazeuse de la matière grasse du lait de vaches. Variations de la composition en acides gras observées en Belgique, Le lait, $n^{\circ}$ 449-450, 603-619.

HADORN (H.), Zürcher (K.) (1969). - Jahreszeitliche Schwankungen der Buttersäure und Capronsäure-Gehalte sowie der Halbmikro-Buttersäurezahl und Gesamtzahl von Butterfett. Mitt. Gebiete Lebensmittelunters. Hyg., 60, 466-489.

HADORN (H.), ZürcheR (K.) (1970). - Die Fettsäure-Verteilung von Butterfett. Dtsch. Lebensmittel-Rundschau, 66, 249-253.

Hall (A. J.) (1970). - Seasonal and regional variations in the fatty acid composition of milk fat. Dairy Ind., 35, 20-24.

HANSEN (R. P.), SHORLAND (F.B.) (1952). - Seasonal variations in fatty acid composition of New-Zealand butterfat. Biochem. J., 52, 207.

Hutton (K.), Seeley (R. C.), Armstrong (D. G.) (1969). - The variations throughout a year in the fatty acid composition of milk fat from two dairy herds. J. Dairy Res., 36, 103-113.

Huyghebeart (A.), Hendrickx (H.) (1970 a). - The relation between the fatty acid composition and the iodine value and refractive index of butterfat. Milchwis., $25,506-510$.

Huyghebeart (A.), Hendrickx (H.) (1970 b). - Onderzoekingen over Belgisch Botervet. I. Inleiding en metoden : De Veresteringsmetode. Gent., 35, 1075-1085.

Huyghebeart (A.), Hendrickx (H.) (1971). - Studies on Belgian butterfat. 3. The fatty acid composition. Milchwis., 26, 613-617.

JAMES (A. T.), MARTIN (A. J. P.) (1952). - Gas-liquid partition chromatography : The separation and microestimation of volatile fatty acids from formic acid to dodecanoïc acid. Biochem. J., 50, 679.

JANOSCHEK (A.), METIN (M.) (1968). - Das Fettsäurespektrum von deutscher Winter und Sommerbutter, türkischer und italienischer Butter, sowie von deutscher Margarine. Milchwis, 23, 29-33.

Keeney (M.), Katz (I.), Allison (M. J.) (1962), - On the probable origin of some milk fat acids in rumen microbial lipids. J. Amer. Oil Chem. Soc., 39, 198-201. 
Kufferath (J.) (1968), - Falsification industrielle du beurre en Belgique. Ann. Fals. Exp. Chim., $\mathbf{n}^{\circ} 679,1-4$.

Kuzdzal-Savoie (S.) (1956). - Influence du stade de lactation sur les caractéristiques de la matière grasse du beurre. Ann. Techn., I.N.R.A., 5, 1-30.

KuzDzal-Savoie (S.), KuzDzal (W.) (1961). - Influence de la mise à l'herbe des vaches laitières sur les indices de la matière grasse du beurre et sur les teneurs en différents acides gras polyinsaturés. Ann. Biol. anim. Biochim. Biophys., 1, 47-69.

Kuzdzal-Savoie (S.), Kuzdzal (W.) (1961). - Les acides polyinsaturés du beurre. Influence de la saison. Ann. Technol. Agric., 10, 73-80.

Kuzdzal-Savoie (S.), Paquot (C.) (1962). - Chromatographie en phase gazeuse et lipochimie. III. Sur l'identification d'un beurre de constitution anormale. Oléagineux, 17, 185-186.

Kuzbzal-Savore (S.), Kuzbzal (W.) (1967 a). - Variations observées dans les proportions relatives des acides gras du lait de vache pendant la période de mise à l'herbe. Congr. Int. Lait., Copenhague, I:1, 270-280.

Kuzdzal-Savoie (S.), KuzDzal (W.) (1967 b). - Les acides gras mineurs du beurre. Le Lait, $\mathrm{n}^{\circ}$ 463-464, 145-155.

Kuzdzal-Savoie (S.), Kuzdzal (W.), LaNglois (D.) (1969). - Contribution à l'étude des acides gras diènes du beurre. Fette Seifen Anstrich., 71, 326-330.

KuzDzal-Savoie (S.), Kuzdzal (W.) (1970). - Etude comparée des acides gras du beurre et de divers corps gras. La Technique Laitière, 684, 15-29.

Matsson (S.), Swartling (P.), Nilson (R.) (1969). - The major fatty acids in whole milk fat and in a fraction obtained by crystallisation from acetone. J. Dairy Res., 36, 169-175.

Metin (M.) (1968). - Fettsäurespektrum türkischer Butter im Vergleich zu deutscher Butter. Milchwiss., 23, 276-279.

Olmedo (R. G.), Gastanaduy (M. C.) (1971). - Contribucion al estudio de las mantequillas espanolas "Composicion en acidos grasos ". Anal. Bromatol., $23,121-200$.

Olmedo (R. G.), Gastanaduy (M. C.) (1971). - Contribucion al estudio de las mantequillas espanolas "Los acidos menores". Anal. Bromatol., 23, 341-356.

Parodr (P. W.) (1970). - Fatty acid composition of australian butter and milk fats. Austr. J. Dairy Technol., 25, 200-205.

PARODI (P.W.) (1972). - Observations on the variation in fatty acid composition of milk fat. Austr. J. Dairy Technol., 27, 90-94.

Patton (S.), McCaRthy (R. D.), Evans (L.), LynN (T.R.) (1960). - Structure and synthesis of milk fat. I. Gas chromatographic analysis. J. Dairy Sci., 43, 1187-1196.

RichaRdSon (T.), McGanN (T.C. A.) (1964). - Fatty acids in Irish butterfat. Irish J. Agric, Res., 3, 151-157.

Roos (J. B.), Versnel (A.), Werdmuller (G. H.) (1963). - Die gaschromatographische Bestimmung der niederen Fettsäuren von Milchfett und deren Anwendung zum Nachweis von Fremdfetten. Kieler Milchw. Forsch., 15, 515-526.

Roos (J. B.) (1964). - Die statistiche Auswertung fet chemischer Kennzahlen. Fette Seifen Anstrich, 66, 182-191.

SENFT (B. von), Klobasa (F.) (1970). - Untersuchungen über das Fettsäurespektrum im Milchfett schwarzbunter Kühe. Milchiwss., 25, 510-514.

Strocchi (A.), Capella (P.), Pallotta (V.), TadDia (M.) (1967). - Il burro profatto in Emilia. Nota II. Composizione in acidi grassi. Ind. Agr., 5, 481-491.

Strocchi (A.), Holman (R. T.) (1971), - Analysis of fatty acids of butterfat. Rev. Ital. Sost. Grasse, 48, 617-622.

Svensen (A.), YstgaARd (O.) (1966), - Die Zusammensetzung der Fettsäuren in norwegischem Butterfett. Int. Dairy Congr., Munich, C, 132-144.

WolfF (J. P.) (1960). - Application de la chromatographie en phase vapeur au contrôle de la pureté des beurres. Ann. Fals. Exp. Chim., 53, 318-325. 